\title{
Effects of Physical Activity on Anthropometric Characteristics in School-Age Children: A Review Study
}

\author{
Ana Lilić, ${ }^{1}$ Emilija Petković, ${ }^{1}$ Siniša Karišik, ${ }^{2}$ Ljiljana Bjelojević, ${ }^{1}$ \\ Novica Gardašević, ${ }^{3}$ Marko Joksimović ${ }^{4}$ \\ University of Nišr ${ }^{1}$ Niš, Serbia \\ University of East Sarajevo², Lukavica, East Sarajevo, Republic of Srpska, Bosnia and Herzegovina \\ Elementary school Dobrislav Đ. Perunović3 ${ }^{3}$ Niksic, Montenegro \\ Institute of Sports and Sports Medicine ${ }^{4}$, Podgorica, Montenegro
}

\begin{abstract}
Background. Today, it is very speculative to talk about the percentage of obese children in the world because these figures change significantly from day to day, and there are even significant differences between respondents in the same country, according to geographical area and place of residence. A constant decline in the level of physical activity of children on a global level can be reported from the age when they start school. Energy intake of food is largely from energy consumption, which leads to an increase in body weight and obesity. Factors of overweight and obesity are associated with a sensitive comparison of health: a significantly increased risk of diabetes, disorders of the locomotor system, and increased social and psychological rights.

Methods and results. During the systematization of the research on the topic, the aim of the paper was to determine whether the influence of physical activity on anthropometric parameters in school-age children is reviewed by previous research. Nineteen types of studies were collected. The following electronic databases were used to search the literature: MEDLINE, SCOPUS, Web of Science SPORTS DISCUS in the period from 2004 to 2016. The respondents included in the research were school-age children.

Conclusion. The positive impact of various physical activity programs is aimed at reducing the parameters of anthropometric characteristics and obesity prevention.
\end{abstract}

Keywords: physical activity, school program, morphology, anthropomeric data, exercises.

\section{INTRODUCTION}

$\mathrm{R}$ esearch across the European region shows a low level of overall physical activity of many populations. More than 14 million children are overweight, and 3 million are obese (World Health Organization, 2006). The results of longitudinal studies indicate that a decrease in physical activity begins as early as age 9 (Hoos, Plasqui, Gerver, \& Westerterp, 2003; Wickel, Eisenmann, \& Welk, 2009). The data obtained show an acute decline in physical activity at all ages over the last few decades (Currie et al., 2004). Across Europe, only one-third of the surveyed school children are physically active at the level recommended by organizations and institutions involved in the promotion of physical exercise (Currie et al., 2004). Children spend more time indoors than ever before. Lack of physical activity and weight gain are also associated with significant health problems in the population of children and adolescents, and thus physical activity is an important factor in reducing the risk of morbidity and mortality in adulthood (Weiss \& Caprio, 2005). Overweight and obesity today represent a new global challenge for public health (Hajmer, 2010).

Obesity is often the result of reduced physical activity, i.e., reduced physical exercise and aerobic fitness (Knöpfli et al., 2008). Acquiring and increasing knowledge and competence in the 
field of physical activity, according to Stodden et al. (2008), is the basis for further physical activity in adulthood. Decreased level of physical activity is associated with an increased rate of obesity adiposity (Lioret, Maire, Volatier, \& Charles, 2007; Riddoch et al., 2004) and decreased values of aerobic and anaerobic capacity (Lafortuna, Fumagalli, Vangeli, \& Sartorio, 2002).

The integration between the progression of obesity and declining levels of physical activity highlights the need for research examining the effects of physical activity. Anthropometric characteristics are one of the ways in which obesity can be tested. The thickness of skin folds represents better parameters and is a better indicator in the assessment of obesity in children in relation to the values of body fat index (Nooyens et al., 2007). Yin et al. (2005) obtained the results which are said to be an additional program of physical activity that students applied during one school year and it had a positive effect on their body composition, i.e. anthropometric data and values that indicated cardiovascular diseases. A program of physical activity lasting 10 weeks leads to the regulation of the body composition of obese children of younger school age (Riddiford-Harland et al., 2016). The Dutch obesity prevention program for school-age children has had beneficial effects on subcutaneous adipose tissue values in both boys and girls (Singh, Chinapaw, Brug, \& Van Mechelen, 2009). The physical activity program applied after school activities in nine-year-old children shows that subjects who were overweight significantly reduced the results in subscapular subcutaneous adipose tissue as well as the body fat index (Messiah et al., 2015). Also, additional physical activity that was applied twice a week for one year, in addition to standard school hours, showed effects on anthropometric values and physical abilities (Kriemler et al., 2010). Longitudinal studies using a physical activity program, alone or with an additional diet program, have shown positive effects on anthropometric characteristics (Deforche et al., 2003; Korsten-Reck et al., 2007; Lazaar et al., 2007; Wong et al., 2008). However, there are studies in which the program of physical activity lasting 28 weeks did not show statistical differences in the values of subcutaneous adipose tissue in boys and girls aged 9 and 10 years (Aguilar et al., 2010). Several review studies have found that a six-month physical activity program in children 6 to 10 years of age did not show positive changes in anthropometric characteristics, but did positively in terms of children's aerobic and anaerobic abilities (Dobbins, Husson, DeCorby, \& LaRocca, 2013; Harris, Kuramoto, Schulzer, \& Retallack, 2009). An extracurricular program lasting four weeks in a study by Matvienko and Ahrabi-Fard, (2010) conducted on first graders showed effects in physical fitness and motor skills, but not anthropometric characteristics. Today, it is very speculative to talk about the percentage of obese children in the world, because these figures change significantly from day to day, and there are even significant differences between respondents in the same country, according to geographical area and place of residence. A constant decline in the level of physical activity of children on a global level can be reported from the age when they start school (Tremblay et al., 2015). Monitoring the nutritional status of children in primary school age is necessary in order to prevent obesity, and thus preserve human health at an early school age. Children who are obese or who have increased body weight are more prone to reduced physical activity, or reduced physical activity is the cause of weight gain (Planinsec \& Matejek, 2004).

The aim of this study was to determine whether there is an impact of physical activity on anthropometric parameters in school-age children by reviewing previous research.

\section{METHODS}

The following electronic databases were used to search the literature: MEDLINE, SCOPUS, Web of Science SPORTS DISCUS in the period from 2004 to 2016. The search was performed using the following keywords (alone or in combination): physical activity, school program, morphology, obesity, children, disorder. The search strategy was modified for each electronic database, where possible, in order to increase sensitivity. All titles and abstracts are reviewed for potential papers to be included in the systematic review. Also, the lists of references of previous review and original research were reviewed. The selection of works was performed on the basis of certain criteria. The analysis included papers that met the defined criteria for inclusion and exclusion from the research. 
Table 1. Criteria for inclusion and exclusion of respondents

\begin{tabular}{|c|c|}
\hline Inclusion criteria & Exclusion criteria \\
\hline 1. effects of physical activity program & $\begin{array}{l}\text { 1. papers examining the relationship between physical activity and anthropo- } \\
\text { metric parameters }\end{array}$ \\
\hline 2. longitudinal research & 2. research articles written in a language other than English \\
\hline 3. research in English & 3. children older than 14 years \\
\hline 4. school-age children & $\begin{array}{l}\text { 4. effects of physical activity program (sports activities) with children who } \\
\text { practice some sports }\end{array}$ \\
\hline
\end{tabular}
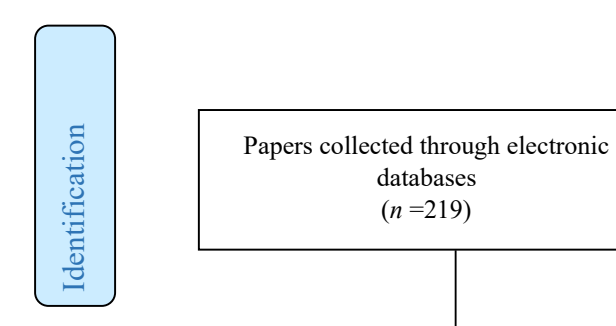

Additional records identified through other sources $(n=17)$
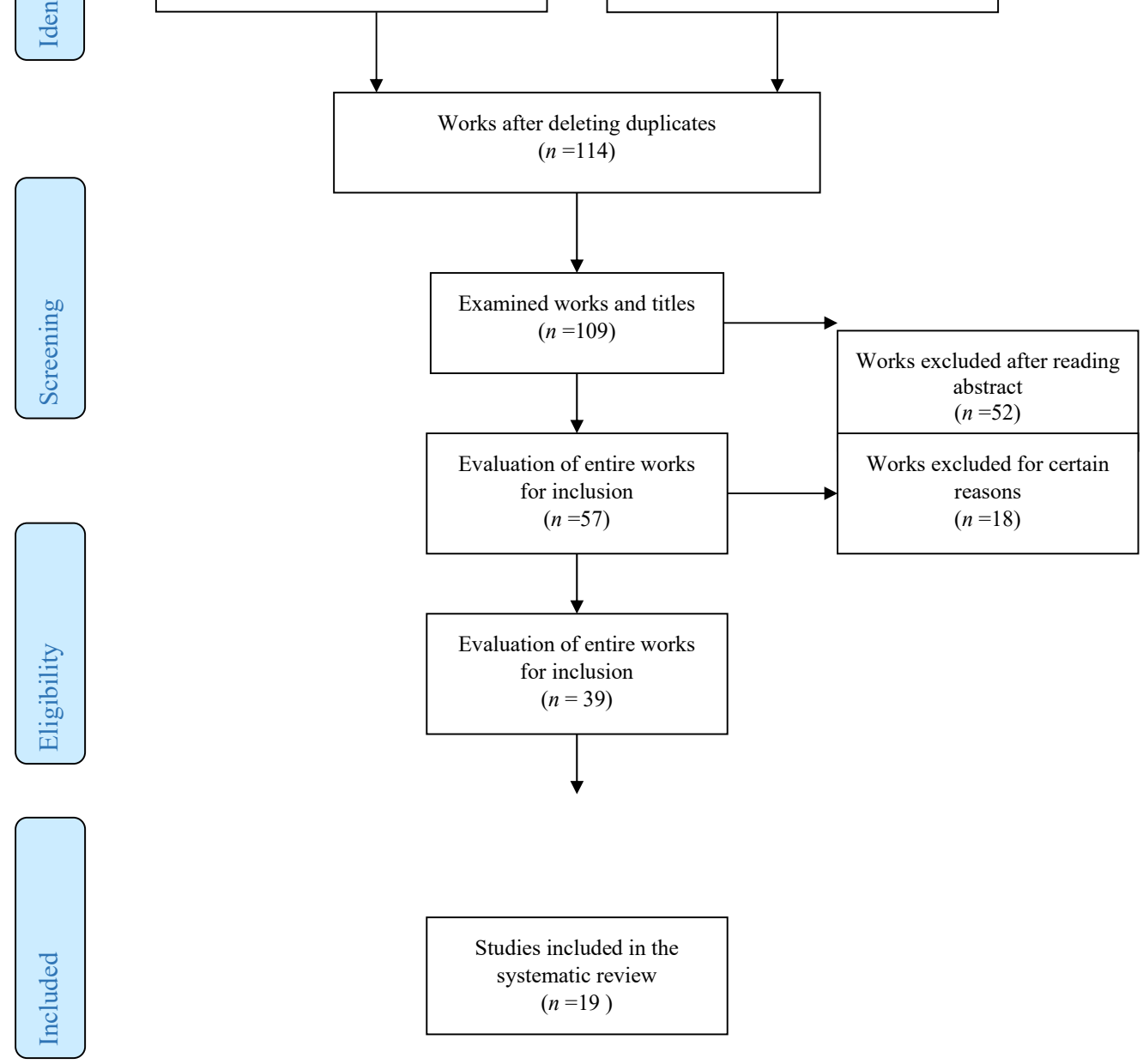

Studies included in the systematic review $(n=19)$

Figure 1. Schematic procedure for the collection, analysis and elimination of works (Moher, Liberati, Tetzlaff, Altman, \& The PRISMA Group, 2010) 
Ana Lilić, Emilija Petković, Siniša Karišik, Ljiljana Bjelojević, Novica Gardašević, Marko Joksimović EFFECTS OF PHYSICAL ACTIVITY ON ANTHROPOMETRIC CHARACTERISTICS IN SCHOOL-AGE CHILDREN: A REVIEW STUDY

\begin{tabular}{|c|c|c|c|c|c|c|c|c|c|}
\hline \multirow[b]{2}{*}{$\begin{array}{c}\text { The first } \\
\text { author } \\
\text { name, } \\
\text { year }\end{array}$} & \multicolumn{3}{|c|}{ Respondents } & \multicolumn{6}{|c|}{ Experimental program } \\
\hline & $\begin{array}{l}\text { No. } \\
\text { respondents }\end{array}$ & Years & Sex & $\begin{array}{l}\text { Exercise } \\
\text { program }\end{array}$ & Frequency & Intensity & $\begin{array}{l}\text { Variables for } \\
\text { estimating } \\
\text { anthropometric } \\
\text { characteristics }\end{array}$ & Results & Conclusion \\
\hline $\begin{array}{l}1 . \\
\text { Park, J. } \\
(2012)\end{array}$ & 29 & $12.2 \pm 1$ & $\mathrm{M}-\mathrm{F}$ & $\begin{array}{c}\mathrm{C} \\
\mathrm{E}(\mathrm{A} \text { and } \mathrm{P})\end{array}$ & $\begin{array}{l}3 \text { times a } \\
\text { week } \\
12 \text { weeks }\end{array}$ & $\begin{array}{c}50 \%-70 \% \\
\text { MHR }\end{array}$ & $\begin{array}{l}\text { Waist } \\
\text { circumference }\end{array}$ & $\mathrm{E}$ and $\mathrm{C}=\uparrow \uparrow$ & $\begin{array}{l}\text { The exercise program } \\
\text { significantly affected } \\
\text { the reduction of waist } \\
\text { circumference. }\end{array}$ \\
\hline $\begin{array}{l}2 . \\
\text { Nassis, G. } \\
(2005)\end{array}$ & 74 & $13.05 \pm 1.75$ & $\mathrm{M}-\mathrm{F}$ & $\mathrm{E}_{1,2}$ (Games) & $\begin{array}{l}3 \text { times a } \\
\text { week } \\
12 \text { weeks }\end{array}$ & $150 \mathrm{HR} / \min$ & $\begin{array}{l}\text { Waist } \\
\text { circumference, } \\
\text { sum of seven skin } \\
\text { folds }\end{array}$ & $\begin{array}{l}E_{1,2} \text { (Waist circum- } \\
\text { ference) }=\uparrow \\
E_{1,2} \text { (sum of seven } \\
\text { skin folds) }=\leftrightarrow\end{array}$ & $\begin{array}{l}\text { The applied game pro- } \\
\text { gram had positive effects } \\
\text { on waist circumference, } \\
\text { but not on sum of seven } \\
\text { skin folds. }\end{array}$ \\
\hline $\begin{array}{l}3 . \\
\text { Yin, Z. } \\
(2005)\end{array}$ & 601 & 8.7 & M-F & $\begin{array}{c}\text { C } \\
\text { E (Games) }\end{array}$ & $\begin{array}{l}2 \text { times a } \\
\text { week } \\
8 \text { Months }\end{array}$ & $149 \mathrm{HR} /_{\min }$ & $\begin{array}{l}\text { Waist } \\
\text { circumference }\end{array}$ & $\mathrm{E}$ and $\mathrm{C}=\leftrightarrow$ & $\begin{array}{l}\text { The application of games } \\
\text { in the aerobic zone did } \\
\text { not give a statistically } \\
\text { significant difference } \\
\text { between } E \text { and } C \text { in waist } \\
\text { circumference. }\end{array}$ \\
\hline $\begin{array}{l}4 . \\
\text { Vizcaíno, } \\
\text { V. (2008) }\end{array}$ & 1119 & $9.4 \pm 0.7$ & M-F & $\begin{array}{l}\mathrm{M}_{\mathrm{E}, \mathrm{C}}(\mathrm{A} \text { and } \mathrm{P}) \\
\mathrm{F}_{\mathrm{E}, \mathrm{C}}(\mathrm{A} \text { and } \mathrm{P})\end{array}$ & $\begin{array}{l}3 \text { times a } \\
\text { week } \\
24 \text { weeks }\end{array}$ & $\begin{array}{c}\text { Accelerom- } \\
\text { eter1345 } \\
\text { steps per } \\
\text { workout }\end{array}$ & $\begin{array}{l}\text { Skin folds of the } \\
\text { triceps }\end{array}$ & $\begin{array}{l}\mathrm{M}_{\mathrm{E}, \mathrm{C}}=\leftrightarrow \\
\mathrm{F}_{\mathrm{E}, \mathrm{C}}=\leftrightarrow\end{array}$ & $\begin{array}{l}\text { No differences were } \\
\text { shown in either boys or } \\
\text { girls in the skin folds of } \\
\text { the triceps after the ap- } \\
\text { plication of the training } \\
\text { program. }\end{array}$ \\
\hline $\begin{array}{l}5 . \\
\text { Riddiford- } \\
\text { Harland, } \\
\text { D. (2016) }\end{array}$ & 34 & $8.5 \pm 1.4$ & M-F & $\begin{array}{c}\mathrm{C}_{1,2} \\
\mathrm{E}_{1,2} \text { (Jumping, } \\
\text { crawling, run- } \\
\text { ning, skipping) }\end{array}$ & $\begin{array}{l}3 \text { times a } \\
\text { week } \\
10 \text { weeks }\end{array}$ & & $\begin{array}{l}\text { Foot length } \\
\text { Foot width }\end{array}$ & $\begin{array}{l}E_{1,2}(\text { Foot length }) \\
=\uparrow \\
C_{1,2}(\text { Foot length }) \\
=\uparrow \\
E_{1,2}(\text { Foot width }) \\
=\leftrightarrow \\
C_{1,2}(\text { Foot width }) \\
=\leftrightarrow\end{array}$ & $\begin{array}{l}\text { It was determined that } \\
\text { there are no differences } \\
\text { between } \mathrm{E} 1,2 \text { and } \mathrm{C} 1,2 \\
\text { in foot width, while } \\
\text { in foot length there is. } \\
\text { However, the authors } \\
\text { found that } \mathrm{K} \text { has a } \\
\text { higher pressure on the } \\
\text { foot itself and therefore } \\
\text { the risks are higher. }\end{array}$ \\
\hline $\begin{array}{l}6 . \\
\text { Zrnzević, } \\
\text { N. (2016) }\end{array}$ & 88 & 7 & $\mathrm{M}-\mathrm{F}$ & $\begin{array}{l}\mathrm{E}_{1,2}(\text { effects of } \\
\text { physical educa- } \\
\text { tion teaching) }\end{array}$ & $\begin{array}{l}3 \text { times a } \\
\text { week } \\
6 \text { Months }\end{array}$ & & $\begin{array}{l}\text { Arm length, leg } \\
\text { length, shoulder } \\
\text { width, pelvic } \\
\text { width, wrist } \\
\text { width, chest } \\
\text { circumference, } \\
\text { upper arm cir- } \\
\text { cumference, thigh } \\
\text { circumference, } \\
\text { skin folds of the } \\
\text { triceps, skin folds } \\
\text { subscapularis, } \\
\text { abdomen skinfold }\end{array}$ & $\begin{array}{l}\mathrm{E}_{1,2} \text { (Arm length, } \\
\text { leg length, shoul- } \\
\text { der width, pelvic } \\
\text { width, wrist width, } \\
\text { chest circumfer- } \\
\text { ence, upper arm } \\
\text { circumference, } \\
\text { thigh circumfer- } \\
\text { ence, }=\uparrow \uparrow \\
\mathrm{E}_{1,2}(\mathrm{skin} \text { folds of } \\
\text { the triceps, skin } \\
\text { folds subscapu- } \\
\text { laris, abdomen } \\
\text { skinfold)= } \leftrightarrow\end{array}$ & $\begin{array}{l}\text { Physical education class- } \\
\text { es at the final measure- } \\
\text { ment had effects on arm } \\
\text { length, leg length, shoul- } \\
\text { der width, wrist width, } \\
\text { chest circumference, up- } \\
\text { per arm circumference, } \\
\text { thigh circumference in } \\
\text { relation to the initial } \\
\text { measurement, while no } \\
\text { statistically significant } \\
\text { data were shown in the } \\
\text { variables skin folds of } \\
\text { the triceps, skin folds } \\
\text { subscapularis, abdomen } \\
\text { skinfold. }\end{array}$ \\
\hline $\begin{array}{l}7 . \\
\text { Thivel, D. } \\
\text { (2011) }\end{array}$ & 57 & $6-10$ & M-F & $\begin{array}{c}\mathrm{C}_{\mathrm{N}, \mathrm{O}} \\
\mathrm{E}_{\mathrm{N}, \mathrm{O}}(\mathrm{P}, \text { Coordi- } \\
\text { nation, speed, } \\
\mathrm{A}) \\
\mathrm{C}_{1,2} \\
\mathrm{E}_{1,2}\end{array}$ & $\begin{array}{l}2 \text { times a } \\
\text { week } \\
6 \text { Months }\end{array}$ & $\begin{array}{l}\text { Moderate } \\
\text { intensity }\end{array}$ & $\begin{array}{l}\text { Waist circumfer- } \\
\text { ence, sum of four } \\
\text { skin folds }\end{array}$ & $\begin{array}{l}\mathrm{C}_{\mathrm{N}, \mathrm{O}} \text { (Waist cir- } \\
\text { cumference, sum } \\
\text { of four skin folds) } \\
=\leftrightarrow \\
\mathrm{C}_{1,2} \text { (Waist cir- } \\
\text { cumference, sum } \\
\text { of four skin folds) } \\
=\leftrightarrow \\
\mathrm{E}_{1,2} \text { (Waist circum- } \\
\text { ference) }=\uparrow \\
\mathrm{E}_{1,2} \text { (sum of four } \\
\text { skin folds) }=\uparrow \uparrow\end{array}$ & $\begin{array}{l}\text { The application of } \\
\text { programs } \mathrm{P} \text {, Coordina- } \\
\text { tion, speed and A gave } \\
\text { positive effects on waist } \\
\text { circumference and sum } \\
\text { of four skin folds. }\end{array}$ \\
\hline
\end{tabular}




\begin{tabular}{|c|c|c|c|c|c|c|c|c|c|}
\hline \multirow[b]{2}{*}{$\begin{array}{l}\text { The first } \\
\text { author } \\
\text { name, } \\
\text { year }\end{array}$} & \multicolumn{3}{|c|}{ Respondents } & \multicolumn{6}{|c|}{ Experimental program } \\
\hline & $\begin{array}{l}\text { No. } \\
\text { respondents }\end{array}$ & Years & Sex & $\begin{array}{l}\text { Exercise } \\
\text { program }\end{array}$ & Frequency & Intensity & $\begin{array}{l}\text { Variables for } \\
\text { estimating } \\
\text { anthropometric } \\
\text { characteristics }\end{array}$ & Results & Conclusion \\
\hline $\begin{array}{l}8 . \\
\text { Messiah, } \\
\text { S. }(2015)\end{array}$ & 349 & 8,9 & $\mathrm{M}-\mathrm{F}$ & $\begin{array}{l}\mathrm{O}_{1,2} \text { (Games) } \\
\mathrm{N}_{1,2} \text { (Games) }\end{array}$ & $\begin{array}{l}2 \text { times a } \\
\text { week } \\
10 \\
\text { Months }\end{array}$ & $\begin{array}{l}\text { Moderately } \\
\text { high inten- } \\
\text { sity }\end{array}$ & $\begin{array}{l}\text { Waist } \\
\text { circumference, } \\
\text { skin folds of the } \\
\text { triceps, skin folds } \\
\text { biceps, skin folds } \\
\text { subscapularis, } \\
\text { skin folds } \\
\text { suprailiac, sum of } \\
\text { four skin folds }\end{array}$ & $\begin{array}{l}\mathrm{N}_{1,2} \text { (Waist cir- } \\
\text { cumference, skin } \\
\text { folds biceps)= } \\
\mathrm{N}_{1,2} \text { (skin folds of } \\
\text { the triceps, sum } \\
\text { of four skin folds) } \\
=\uparrow \uparrow \\
\mathrm{N}_{1,2} \text { (skin folds } \\
\text { subscapularis, } \\
\text { skin folds su- } \\
\text { prailiac) }=\uparrow \\
\mathrm{O}_{1,2} \text { (Waist cir- } \\
\text { cumference, } \\
\text { skin folds of the } \\
\text { triceps, skin folds } \\
\text { biceps, skin folds } \\
\text { suprailiac, sum of } \\
\text { four skin folds) } \\
=\leftrightarrow \\
\mathrm{O}_{1,2} \text { (skin folds } \\
\text { subscapularis) } \\
=\uparrow \uparrow\end{array}$ & $\begin{array}{l}\text { In the group of nor- } \\
\text { mally fed students, the } \\
\text { program of the game } \\
\text { program showed better } \\
\text { results in anthropometric } \\
\text { characteristics than in } \\
\text { the group of obese. }\end{array}$ \\
\hline $\begin{array}{l}9 . \\
\text { Lazaar, } \\
\text { N. (2007) }\end{array}$ & 425 & $6-10$ & $\mathrm{M}-\mathrm{F}$ & $\begin{array}{c}\mathrm{C}_{\mathrm{N}, \mathrm{O}} \\
\mathrm{E}_{\mathrm{N} 1, \mathrm{O} 1} \text { (Games, } \\
\text { coordination) } \\
\mathrm{E}_{\mathrm{N} 2, \mathrm{O} 2}\end{array}$ & $\begin{array}{l}2 \text { times a } \\
\text { week } \\
6 \text { Months }\end{array}$ & $\begin{array}{l}\text { Moderately } \\
\text { high inten- } \\
\text { sity }\end{array}$ & $\begin{array}{l}\text { Waist } \\
\text { circumference, } \\
\text { sum of four skin } \\
\text { folds }\end{array}$ & $\begin{array}{l}\mathrm{E}_{\mathrm{N} 1,01}-\mathrm{E}_{\mathrm{N} 2, \mathrm{O} 2} \\
\text { (Waist circumfer- } \\
\text { ence, sum of four } \\
\text { skin folds) }=\uparrow \uparrow\end{array}$ & $\begin{array}{l}\text { The application of physi- } \\
\text { cal exercise significantly } \\
\text { influenced the anthropo- } \\
\text { metric characteristics of } \\
\text { waist circumference and } \\
\text { sum of four skin folds. }\end{array}$ \\
\hline $\begin{array}{l}10 . \\
\text { Kriemler, } \\
\text { S. }(2010)\end{array}$ & 502 & $6.9-11.3$ & $\mathrm{M}-\mathrm{F}$ & $\begin{array}{c}\mathrm{C}_{1,2} \\
\mathrm{E}_{1,2} \\
\text { (P,Coordination, } \\
\text { jumping) }\end{array}$ & $\begin{array}{l}2 \text { times a } \\
\text { week } \\
6 \text { Months }\end{array}$ & & $\begin{array}{l}\text { Waist } \\
\text { circumference, } \\
\text { sum of four skin } \\
\text { folds }\end{array}$ & $\begin{array}{l}C_{1,2} \text { (Waist circum- } \\
\text { ference) }=\leftrightarrow \\
E_{1,2} \text { (Waist circum- } \\
\text { ference, sum of } \\
\text { four skin folds) } \\
=\leftrightarrow \\
C_{1,2} \text { (sum of four } \\
\text { skin folds) }=\uparrow \uparrow\end{array}$ & $\begin{array}{l}\text { Values of C in the sec- } \\
\text { ond measurement (sum } \\
\text { of four skin folds) }=\uparrow \uparrow \text {, } \\
\text { but there was an increase } \\
\text { in the value compared to } \\
\text { the first measurement. }\end{array}$ \\
\hline $\begin{array}{l}11 . \\
\text { Zorba, E. } \\
\text { (2011) }\end{array}$ & 40 & $11 \pm 1$ & M & $\begin{array}{c}\mathrm{C}_{1,2} \\
\mathrm{E}_{1,2}(\mathrm{~A}, \mathrm{~T})\end{array}$ & $\begin{array}{l}3 \text { times a } \\
\text { week } \\
12 \\
\text { Months }\end{array}$ & $\begin{array}{c}60 \%-65 \% \\
\text { MHR }\end{array}$ & $\begin{array}{l}\text { Chest } \\
\text { circumference, } \\
\text { upper arm } \\
\text { circumference, } \\
\text { lower leg } \\
\text { circumference, } \\
\text { waist } \\
\text { circumference, } \\
\text { hip } \\
\text { circumference, } \\
\text { elbow joint width, } \\
\text { knee joint width }\end{array}$ & $\begin{array}{l}\mathrm{C}_{1,2}(\mathrm{Chest} \text { circum- } \\
\text { ference, arm cir- } \\
\text { cumference, lower } \\
\text { leg circumference, } \\
\text { waist circumfer- } \\
\text { ence, hip circum- } \\
\text { ference, elbow } \\
\text { joint width, knee } \\
\text { joint width) = } \uparrow \uparrow \\
\mathrm{E}_{1,2}(\text { Chest circum- } \\
\text { ference, arm cir- } \\
\text { cumference, lower } \\
\text { leg circumference, } \\
\text { waist circumfer- } \\
\text { ence, hip circum- } \\
\text { ference, elbow } \\
\text { joint width, knee } \\
\text { joint width) = } \uparrow \uparrow\end{array}$ & $\begin{array}{l}\text { The physical activity } \\
\text { program had positive } \\
\text { effects on anthropo- } \\
\text { metric characteristics in } \\
\mathrm{E} \text {, while in } \mathrm{C} \text { there is a } \\
\text { significant difference, } \\
\text { but there was an increase } \\
\text { in all variables }\end{array}$ \\
\hline $\begin{array}{l}12 . \\
\text { Aguilar, } \\
\text { F. (2010) }\end{array}$ & 1044 & $9-10$ & $\mathrm{M}-\mathrm{F}$ & $\begin{array}{l}\mathrm{M}_{\mathrm{E}, \mathrm{C}} \text { (Jumping, } \\
\text { dance) } \\
\mathrm{F}_{\mathrm{E}, \mathrm{C}} \text { (Jumping, } \\
\text { dance) }\end{array}$ & $\begin{array}{l}3 \text { times a } \\
\text { week } \\
28 \\
\text { Months }\end{array}$ & $\begin{array}{l}\text { Accelerom- } \\
\text { eter1345 } \\
\text { steps per } \\
\text { workout }\end{array}$ & $\begin{array}{l}\text { Skin folds of the } \\
\text { triceps }\end{array}$ & $\begin{array}{l}\mathrm{F}_{\mathrm{E}, \mathrm{C}} \text { (Skin folds of } \\
\text { the triceps) }=\uparrow \uparrow \\
\mathrm{M}_{\mathrm{E}, \mathrm{C}} \text { (Skin folds of } \\
\text { the triceps) }=\uparrow \uparrow\end{array}$ & $\begin{array}{l}\text { Physical exercise had a } \\
\text { positive effect on both } \\
\mathrm{M} \text { and F. In subject E, } \\
\text { there was a decrease in } \\
\text { the value in skin folds of } \\
\text { the triceps. }\end{array}$ \\
\hline
\end{tabular}


Ana Lilić, Emilija Petković, Siniša Karišik, Ljiljana Bjelojević, Novica Gardašević, Marko Joksimović EFFECTS OF PHYSICAL ACTIVITY ON ANTHROPOMETRIC CHARACTERISTICS IN SCHOOL-AGE CHILDREN: A REVIEW STUDY

\begin{tabular}{|c|c|c|c|c|c|c|c|c|c|}
\hline \multirow[b]{2}{*}{$\begin{array}{l}\text { The first } \\
\text { author } \\
\text { name, } \\
\text { year }\end{array}$} & \multicolumn{3}{|c|}{ Respondents } & \multicolumn{6}{|c|}{ Experimental program } \\
\hline & $\begin{array}{l}\text { No. } \\
\text { respondents }\end{array}$ & Years & Sex & $\begin{array}{l}\text { Exercise } \\
\text { program }\end{array}$ & Frequency & Intensity & $\begin{array}{l}\text { Variables for } \\
\text { estimating } \\
\text { anthropometric } \\
\text { characteristics }\end{array}$ & Results & Conclusion \\
\hline $\begin{array}{l}13 . \\
\text { Magnus- } \\
\text { son, K. } \\
(2012)\end{array}$ & 321 & $7.3 \pm 0.3$ & $\mathrm{M}-\mathrm{F}$ & $\begin{array}{c}\mathrm{C} \\
\mathrm{E}(\text { additional } \\
\text { classes of } \mathrm{PE} \\
\text { and education) }\end{array}$ & $\begin{array}{l}3 \text { times a } \\
\text { week } \\
2 \text { years }\end{array}$ & & $\begin{array}{l}5 \text { skin folds sum, } \\
\text { waist circumfer- } \\
\text { ence }\end{array}$ & $\mathrm{E}$ and $\mathrm{C}=\leftrightarrow$ & $\begin{array}{l}\text { After two years of ap- } \\
\text { plying an additional hour } \\
\text { of physical education, } \\
\text { E showed better results } \\
\text { than C, but they were not } \\
\text { statistically significant } \\
\text { enough. }\end{array}$ \\
\hline $\begin{array}{l}14 . \\
\text { Bocca, G. } \\
(2012)\end{array}$ & 75 & $3-5$ & M-F & $\begin{array}{c}\text { C } \\
\text { E (Games, } \\
\text { dance) }\end{array}$ & $\begin{array}{l}3 \text { times a } \\
\text { week } \\
12 \\
\text { Months }\end{array}$ & & $\begin{array}{l}\text { Waist circumfer- } \\
\text { ence, hip circum- } \\
\text { ference }\end{array}$ & $\begin{array}{l}\mathrm{E} \text { and } \mathrm{C} \text { (waist } \\
\text { circumference) } \\
=\uparrow \uparrow\end{array}$ & $\begin{array}{l}\text { There are positive effects } \\
\text { of the applied exercise } \\
\text { program. }\end{array}$ \\
\hline $\begin{array}{l}15 . \\
\text { Kain, J. } \\
\text { (2004) }\end{array}$ & 3086 & $10.6 \pm 2.6$ & $\mathrm{M}-\mathrm{F}$ & $\begin{array}{c}\text { C } \\
\text { E (Soccer, } \\
\text { coordination, } \\
\text { volleyball) }\end{array}$ & $\begin{array}{l}3 \text { times a } \\
\text { week } \\
6 \text { Months }\end{array}$ & $\begin{array}{l}\text { Moderately } \\
\text { high inten- } \\
\quad \text { sity }\end{array}$ & $\begin{array}{l}\text { Waist circumfer- } \\
\text { ence, Skin folds } \\
\text { of the triceps }\end{array}$ & $\begin{array}{l}E \text { and } C \text { (Waist cir- } \\
\text { cumference) }=\uparrow \uparrow \\
E \text { and } C \text { (Skin } \\
\text { folds of the tri- } \\
\text { ceps) }=\leftrightarrow\end{array}$ & $\begin{array}{l}\text { The program of physical } \\
\text { activity had a positive } \\
\text { effect on the reduction } \\
\text { of waist circumference } \\
\text { in E. }\end{array}$ \\
\hline $\begin{array}{l}16 . \\
\text { Nemet, } \\
\text { D. }(2005)\end{array}$ & 46 & $10-11$ & M-F & $\begin{array}{c}\text { C } \\
\text { E (Games, } \\
\text { running) }\end{array}$ & $\begin{array}{c}2 \text { times a } \\
\text { week } \\
3 \text { Months }\end{array}$ & & 2 skin fold sum & $\begin{array}{l}E \text { and } C(2 \text { skin } \\
\text { fold sum })=\uparrow\end{array}$ & $\begin{array}{l}\text { There was a decrease in } \\
\text { subcutaneous adipose } \\
\text { tissue in E, while in C } \\
\text { there was an increase in } \\
\text { values. }\end{array}$ \\
\hline $\begin{array}{l}17 . \\
\text { Blüher, } \\
\text { S.(2014) }\end{array}$ & 257 & $10.5-11.4$ & $\mathrm{M}-\mathrm{F}$ & $\mathrm{E}_{1,2}(\mathrm{~A}, \mathrm{P})$ & $\begin{array}{l}2 \text { times a } \\
\text { week } \\
\text { 12Months }\end{array}$ & & $\begin{array}{l}\text { Waist circumfer- } \\
\text { ence }\end{array}$ & $\begin{array}{l}E_{1,2} \text { (waist circum- } \\
\text { ference) }=\uparrow\end{array}$ & $\begin{array}{l}\text { The exercise program } \\
\text { led to statistically sig- } \\
\text { nificant changes between } \\
\text { the first and second } \\
\text { measurements. }\end{array}$ \\
\hline $\begin{array}{l}\text { 18. Kain, } \\
\text { J. (2009) }\end{array}$ & 2039 & $8-12$ & M-F & $\begin{array}{c}C \\
E(A)\end{array}$ & 2 years & & $\begin{array}{l}\text { Waist circumfer- } \\
\text { ence, Skin folds } \\
\text { of the triceps }\end{array}$ & $\begin{array}{l}\mathrm{E} \text { and } \mathrm{C} \text { (Waist } \\
\text { circumference, } \\
\text { Skin folds of the } \\
\text { triceps) }=\leftrightarrow\end{array}$ & $\begin{array}{l}\text { Aerobic exercises did } \\
\text { not show statistically } \\
\text { significant differences } \\
\text { between E and C. }\end{array}$ \\
\hline $\begin{array}{l}19 . \\
\text { Pelemiš, } \\
\text { V. (2016) }\end{array}$ & 211 & $6-7$ & $\mathrm{M}-\mathrm{F}$ & $\begin{array}{c}\mathrm{C} 1 \\
\mathrm{C} 2 \\
\mathrm{E} \text { (Directed mo- } \\
\text { tor activities) }\end{array}$ & $\begin{array}{l}2 \text { times a } \\
\text { week } \\
24 \\
\text { Months }\end{array}$ & & $\begin{array}{l}\text { Chest circumfer- } \\
\text { ence, upper arm } \\
\text { circumference, } \\
\text { lower leg circum- } \\
\text { ference, abdo- } \\
\text { men skinfold, } \\
\text { skin folds of the } \\
\text { triceps, skin folds } \\
\text { subscapularis }\end{array}$ & $\begin{array}{l}\text { E and C1 (Upper } \\
\text { arm circumfer- } \\
\text { ence) }=\uparrow \uparrow \\
\text { E and C1,C2 } \\
\text { (Lower leg cir- } \\
\text { cumference) }=\uparrow \uparrow \\
\text { E and C2 (Abdo- } \\
\text { men skinfold) = } \uparrow \\
\text { E and C2 (skin } \\
\text { folds of the tri- } \\
\text { ceps) }=\uparrow \uparrow \\
\text { E and C1,C2 } \\
\text { (Chest circumfer- } \\
\text { ence, skin folds } \\
\text { subscapularis) } \\
=\leftrightarrow\end{array}$ & $\begin{array}{l}\text { There is an influence of } \\
\text { the experiment which is } \\
\text { reflected in the reduced } \\
\text { values of anthropometric } \\
\text { characteristics. }\end{array}$ \\
\hline
\end{tabular}

Notes: M-men; F-women; C-control group; E-experimental group, MHR-maximal heart rate; A- aerobic activities; P-power; I-games; HR-heart rate beats; $\mathrm{E}_{1,2}$-experimental group first and second measurements; $\uparrow \uparrow-\mathrm{p}<.01 ; \uparrow-\mathrm{p}<.05 ; \leftrightarrow-$ no statistical significance; $\mathrm{M}_{\mathrm{E}, \mathrm{C}}-\mathrm{male}$ experimental and control group; $\mathrm{F}_{\mathrm{E}, \mathrm{C}}$-women experimental and control group; $\mathrm{C}_{\mathrm{N}, \mathrm{O}-}$ normal fed and obese control group ; $\mathrm{E}_{\mathrm{N}, \mathrm{O}^{-}}$normal fed and obese control group experimental group; $\mathrm{C}_{1,2^{-}}$ control group $1^{\text {st }}$ and $2^{\text {nd }}$ measuring; $\mathrm{O}_{1,2^{-}}$obese $1^{\text {st }}$ and $2^{\text {nd }}$ measuring; $\mathrm{N}_{1,2^{-}}$normal fed 1 st and $2^{\text {nd }}$ measuring; $\mathrm{E}_{\mathrm{N} 1, \mathrm{O} 1^{-}}$normal fed and obese experimental group $1^{\text {st }}$ measuring; $\mathrm{E}_{\mathrm{N} 2, \mathrm{O}^{-}}$- normal fed and obese experimental group 2nd $2^{\text {nd }}$ measuring; PE-physical education; C1- control group 1; C2- control group 2. 


\section{RESULTS}

After searching the available scientific databases, 114 papers were collected, which were originally selected by search. After reviewing these papers due to the subject and goal of certain papers that do not include the subject and goal of our research, based on exclusion criteria, we had to reject 94 papers because they did not deal with the impact of a physical activity program on anthropometric characteristics. The total number of studies that are included in the systematization is 19 , where boys and girls are included, and the research is related to the effects of physical exercise programs on anthropometric characteristics. The subjects included in the study were school-age children. The programs of physical activity that were taken into the analysis of the review work are: activities that include the elementary type of locomotion (jumping, crawling, running, skipping), strength development exercises, coordination exercises, speed exercises, ball games (football, basketball, volleyball), dance, as well as the effects of physical education classes themselves. The duration of the experimental program was at least ten weeks (Riddiford-Harland et al., 2016), while the longest program lasted two years (Kain et al., 2009; Magnusson et al., 2012). The frequency of training was 2 to 3 times a week. Pelemis (2016); Blüher et al. (2014); Nemet et al. (2005); Kriemler et al. (2010); Lazaar et al. (2007); Messiah et al. (2015); Thivel et al. (2011); Yin et al. (2005) in these studies the physical activity program was applied twice a week, while in the studies (Cain et al., 2004; Bocca, Corpeleijn, Stolk, \& Sauer, 2012; Aguilar et al., 2010; Magnussonet et al., 2012; Zorba, Cengiz, \& Karacabey, 2011; Zrnzevic \& Zrnzevic, 2016; Riddiford-Harland et al., 2016; Vizcainoet et al., 2008; Nassis et al., 2005; Park et al., 2012) frequency physical activity program was three times a week. The intensity of physical activity was moderate intensity (Zorba et al., 2011; Thivel et al., 2011; Nassis et al., 2005; Park et al., 2012; Yin et al., 2005) to moderately high intensity (Kain et al., 2004; Messiah et al., 2015; Lazaar et al., 2007).

\section{DISCUSSION}

The aim of this study was to determine whether there is an impact of physical activity on anthropometric parameters in school-age children by reviewing previous research. When it comes to the effects of the applied program of physical activity, application of games, duration of eight months and application twice a week, it was found that there was no statistically significant difference between the experimental and control group in values of waist circumference (Yin et al., 2005). Also, Kriemler et al. (2010) found that the program lasting six months with a frequency of twice a week did not show significant differences in the first and second measurements in the experimental and control groups in the parameters of waist circumference and total value of four skin folds. Physical activity did not give significant results on anthropometric characteristics. However, in several studies the application of different types of physical activity (ball games, coordination, strength) twice a week showed positive changes (Pelemis, 2016; Thivel et al., 2011; Lazaar et al., 2007; Messiah et al., 2015). Pelemis (2016) obtained results in which the experimental group after the program of motor activities significantly differed from the control group in upper arm circumference, lower leg circumference, abdomen skinfold, and skin folds of the triceps $(p<.01)$. When it comes to the application of games and coordination exercises lasting six months, waist circumference and the total value of the four skin folds in the first and second measurements indicated significant changes in both obese and normal weight students $(p<.01)$ (Lazaar et al., 2007). The same anthropometric characteristics were found in the study of Thivel et al. (2011) in the group of students who applied the program of strength, speed, coordination and aerobic exercise, as well as differences in the initial and final measurements (waist circumference $(p<.05)$ and the sum of four skin folds $(p<.01)$. The application of games influenced the difference between the first and second measurements in skin folds subscapularis $(p<.05)$ in obese subjects and skin folds of the triceps, sum of four skin folds $(p<.05)$, skin folds subscapularis, skin folds suprailiac $(p<.01)$ in normal weight students (Messiah et al., 2015). Vizcaíno et al. (2008), Magnusson et al. (2012), Kain et al. (2009) with the frequency of physical activity three times a week found no effects on anthropometric characteristics. Foot lengths of the first and second measurements were significant in both the experimental group and the control group $(p<.05)$ (Riddiford-Harland et al., 2016). The effects of physical education classes (Zrnzević \& Zrnzević, 2016) lasting six months with the frequency of three times a week had a positive 
effect on arm length, leg length, shoulder width, pelvic width, wrist width, chest circumference, upper arm circumference, thigh circumference between initial and final measurements $(p<.01)$, while in the variables such as skin folds of the triceps, skin folds subscapularis, abdomen skinfold no effects of physical education were found. Activities such as jumping and dancing show that there was a difference between the control group and the group that applied the physical activity program in skin folds of the triceps $(p<.01)$ in both boys and girls (Aguilar et al., 2010). However, Kain et al., 2004 did not show statistically significant differences in skin folds of the triceps, while the differences between the control and experimental groups in waist circumference were observed $(p<.01)$. Also, these differences in waist circumference $(p<.01)$ can be seen in other studies (Bocca et al., 2012).

Middle school students whose planned physical activity program had a frequency of twice a week showed that there were differencesin anthropometric characteristics (Blüher et al., 2014; Nemet et al., 2005). Blüher et al. (2014) found that there was a difference in the initial and final measurements when applying the program of strength exercises and aerobic exercise waist circumference $(p<.05)$. Nemet et al. (2005) found that the exercise program, which included the application of games and running exercises with the duration of three months, showed differences between the control and experimental groups in skin fold sum $(p<.05)$. Studies where experimental treatment lasted for twelve weeks, which included strength exercises, running exercises in games, indicated positive changes in anthropometric characteristics (Nassis et al., 2005; Park et al., 2012; Zorba et al., 2011). According to Zorba et al. (2011), for participants who applied aerobic exercises and running exercises, the results in the initial and final measurements differed significantly in chest circumference, upper arm circumference, lower leg circumference, waist circumference, hip circumference, elbow joint width, and knee joint width $(p<.01)$. The variable waist circumference in the study of Park et al. (2012) indicated differences $(p<.01)$ between the group of students who participated in the exercise program and the control group of students. Also, Nassis et al. (2005) indicated that there were differences between games in the waist circumference $(p<.05)$, but not in the variable sum of seven skin folds which did not indicate differences.

\section{CONCLUSION}

Based on the reviewed papers, it was determined that physical activity had a positive effect on anthropometric parameters in children of middle school age, but not in children of younger school age. The proposal and conclusion are that, in order to obtain the most accurate results, due to the period of growth and development in which schoolage children are, in addition to anthropometric characteristics, further research also should include indices of body composition to determine the level of changes. Are these changes caused by an increase in muscle mass and loss of fat deposits or is it the other way around?

\section{REFERENCES}

Aguilar, F. S., Martínez-Vizcaíno, V., López, M. S., Martínez, M. S., Gutiérrez, R. F., Martínez, S. S., LopezGarcia, E., \& Rodríguez-Artalejo, F. (2010). Impact of an after-school physical activity program on obesity in children. The Journal of Pediatrics, 157(1), 36-42.doi. org/10.1016/j.jpeds.2009.12.046

Blüher, S., Petroff, D., Wagner, A., Warich, K., Gausche, R., Klemm, T., Wagner, M., \& Keller, A. (2014). The one-year exercise and lifestyle intervention program KLAKS: Effects on anthropometric parameters, cardiometabolic risk factors and glycemic control in childhood obesity. Metabolism, 63(3), 422-430.doi. org/10.1016/j.metabol.2013.11.016

Bocca, G., Corpeleijn, E., Stolk, R. P., \& Sauer, P. J. (2012). Results of a multidisciplinary treatment program in 3-year-old to 5-year-old overweight or obese children: A randomized controlled clinical trial. Archives of Pediatrics
\& Adolescent Medicine, 166(12), 1109-1115.doi:10.1001/ archpediatrics.2012.1638

Weiss, R.\& Caprio, S. (2005). The metabolic consequences of childhood obesity. Best Practice and Research Clinical Endocrinology and Metabolism, 19(3), 405-419.doi.org/10.1016/j.beem.2005.04.009

Currie, C., Roberts, C., Settertobulte, W., Morgan, A., Smith, R., Samdal, O., Rasmussen, B., V., \& World Health Organization. (2004). Young people's health in context: Health Behaviour in School-aged Children (HBSC) study: international report from the 2001/2002 survey (No. EUR/04/5048327). Copenhagen: WHO Regional Office for Europe.

Deforche, B., De Bourdeaudhuij, I., Debode, P., Vinaimont, F., Hills, A. P., Verstraete, S., \& Bouckaert, J. (2003). Changes in fat mass, fat-free mass and aerobic fitness in severely obese children and adolescents 
following a residential treatment programme. European Journal of Pediatrics, 162(9), 616-622.doi: 10.1007/ s00431-003-1247-2

Dobbins, M., Husson, H., DeCorby, K., \& LaRocca, R. L. (2013). School-based physical activity programs for promoting physical activity and fitness in children and adolescents aged 6 to 18. Cochrane Database of Systematic Reviews, (2), 1-149.doi. org/10.1002/14651858.CD007651.pub2

Hajmer, S. (2010). Physical activity and health in Europe. Zagreb: Faculty of Kinesiology.

Harris, K. C., Kuramoto, L. K., Schulzer, M., \& Retallack, J. E. (2009). Effect of school-based physical activity interventions on body mass index in children: A meta-analysis. Canadian Medical Association Journal, 180(7), 719-726. doi.org/10.1503/cmaj.080966 Hoos, M. B., Plasqui, G., Gerver, W. J. M., \& Westerterp, K. R. (2003). Physical activity level measured by doubly labeled water and accelerometry in children. European Journal of Applied Physiology, 89(6), 624-626., doi: 10.1007/s00421-003-0891-6.

Kain, J., Leyton, B., Cerda, R., Vio, F., \& Uauy, R. (2009). Two-year controlled effectiveness trial of a school-based intervention to prevent obesity in Chilean children. Public Health Nutrition, 12(9), 1451-1461. doi: https://doi.org/10.1017/S136898000800428X

Kain, J., Uauy, R., Vio, F., Cerda, R., \& Leyton, B. (2004). School-based obesity prevention in Chilean primary school children: Methodology and evaluation of a controlled study. International Journal of Obesity, 28(4), 483-493.doi:10.1038/sj.ijo.0802611

Knöpfli, B. H., Radtke, T., Lehmann, M., Schätzle, B., Eisenblätter, J., Gachnang, A., Wiederkehr, P., Hammer, J., \& Brooks-Wildhaber, J. (2008). Effects of a multidisciplinary inpatient intervention on body composition, aerobic fitness, and quality of life in severely obese girls and boys. Journal of Adolescent Health, 42(2), 119-127.doi.org/10.1016/j.jadohealth.2007.08.015

Korsten-Reck, U., Kaspar, T., Korsten, K., KromeyerHauschild, K., Bös, K., Berg, A., \& Dickhuth, H. H. (2007). Motor abilities and aerobic fitness of obese children. International Journal of Sports Medicine, 28(09), 762-767.

Kriemler, S., Zahner, L., Schindler, C., Meyer, U., Hartmann, T., Hebestreit, H., Brunner-La Rocca, H. P., van Mechelen, W., \& Puder, J. J. (2010). Effect of school based physical activity programme (KISS) on fitness and adiposity in primary schoolchildren: cluster randomised controlled trial. British Medical Journal, 340, 785793. doi: https://doi.org/10.1136/bmj.c785

Lafortuna, C. L., Fumagalli, E., Vangeli, V., \& Sartorio, A. (2002). Lower limb alactic anaerobic power output assessed with different techniques in morbid obesity. Journal of Endocrinological Investigation, 25(2), 134-141.

Lazaar, N., Aucouturier, J., Ratel, S., Rance, M., Meyer, M., \& Duché, P. (2007). Effect of physical activity intervention on body composition in young children: Influence of body mass index status and gender. Acta
Paediatrica, 96(9), 1321-1325. https://doi.org/10.1111/ j.1651-2227.2007.00426.x

Lioret, S., Maire, B., Volatier, J. L., \& Charles, M. A. (2007). Child overweight in France and its relationship with physical activity, sedentary behaviour and socioeconomic status. European Journal of Clinical Nutrition, 61(4), 509-516.doi:10.1038/sj.ejcn.1602538

Magnusson, K. T., Hrafnkelsson, H., Sigurgeirsson, I., Johannsson, E., \& Sveinsson, T. (2012). Limited effects of a 2-year school-based physical activity intervention on body composition and cardiorespiratory fitness in 7-year-old children. Health education research, 27(3), 484-494.doi.org/10.1093/her/cys049

Matvienko, O., \& Ahrabi-Fard, I. (2010). The effects of a 4-week after-school program on motor skills and fitness of kindergarten and first-grade students. American Journal of Health Promotion, 24(5), 299-303.doi. org/10.4278/ajhp.08050146

Messiah, S. E., Diego, A., Kardys, J., Kirwin, K., Hanson, E., Nottage, R., Ramirez, S., \& Arheart, K. L. (2015). Effect of a park-based after-school program on participant obesity-related health outcomes. American Journal of Health Promotion, 29(4), 217-225.doi. org/10.4278/ajhp.120705-QUAN-327

Moher, D., Liberati, A., Tetzlaff, J., \& Altman, D. G. (2010). Preferred reporting items for systematic reviews and meta-analyses: the PRISMA statement. PLoS Medicine 6(5), 336-341. https://doi.org/10.1371/journal. pmed.1000097

Nassis, G. P., Papantakou, K., Skenderi, K., Triandafillopoulou, M., Kavouras, S. A., Yannakoulia, M., Chrousos, G. P., \& Sidossis, L. S. (2005). Aerobic exercise training improves insulin sensitivity without changes in body weight, body fat, adiponectin, and inflammatory markers in overweight and obese girls. Metabolism, 54(11), 1472-1479.doi.org/10.1016/j.metabol.2005.05.013

Nemet, D., Barkan, S., Epstein, Y., Friedland, O., Kowen, G., \& Eliakim, A. (2005). Short-and long-term beneficial effects of a combined dietary-behavioralphysical activity intervention for the treatment of childhood obesity. Pediatrics, 115(4), 443-449. doi. org/10.1542/peds.2004-2172

Nooyens, A. C., Koppes, L. L., Visscher, T. L., Twisk, J. W., Kemper, H. C., Schuit, A. J., van Mechelen, W., \& Seidell, J. C. (2007). Adolescent skinfold thickness is a better predictor of high body fatness in adults than is body mass index: The Amsterdam Growth and Health Longitudinal Study. The American Journal of Clinical Nutrition, 85(6), 1533-1539.doi.org/10.1093/ ajen/85.6.1533

Park, J. H., Miyashita, M., Kwon, Y. C., Park, H. T., Kim, E. H., Park, J. K., Park, B. K., Yoon, S. R., Chung, J. W., Nakamura, Y., \& Park, S. K. (2012). A 12week after-school physical activity programme improves endothelial cell function in overweight and obese children: a randomised controlled study. BMC Pediatrics, 12(1), 111-120.doi:10.1186/1471-2431-12-111

Pelemiš, V. (2016). Influence of additional physical exercise program on morphological and motor status 
of preschool children. Doctoral dissertation, Novi Sad: Faculty of sport and PE University of Novi Sad.

Planinsec, J., \& Matejek, C. (2004). Differences in physical activity between non-overweight, overweight and obese children. Collegium Antropologicum, 28(2), 747-754.

Riddiford-Harland, D. L., Steele, J. R., Cliff, D. P., Okely, A. D., Morgan, P. J., \& Baur, L. A. (2016). Does participation in a physical activity program impact upon the feet of overweight and obese children? Journal of Science and Medicine in Sport, 19(1), 51-55.doi. org/10.1016/j.jsams.2014.11.008

Riddoch, C. J., Andersen, L. B., Wedderkopp, N., Harro, M., Klasson-Heggebø, L., Sardinha, L. B., Cooper, A. R., \& Ekelund, U. L. F. (2004). Physical activity levels and patterns of 9-and 15-yr-old European children. Medicine \& Science in Sports \& Exercise, 36(1), 86-92.doi: 10.1249/01.MSS.0000106174.43932.92

Rønning, E. (2001). Living conditions of children before and now. Oslo: Statistisk Sentralbyra.

Singh, A. S., Chinapaw, M. J. M., Brug, J., \& Van Mechelen, W. (2009). Process evaluation of a schoolbased weight gain prevention program: The Dutch Obesity Intervention in Teenagers (DOiT). Health Education Research, 24(5), 772-777.doi.org/10.1093/her/cyp011

Stodden, D. F., Goodway, J. D., Langendorfer, S. J., Roberton, M. A., Rudisill, M. E., Garcia, C., \& Garcia, L. E. (2008). A developmental perspective on the role of motor skill competence in physical activity: An emergent relationship. Quest, 60(2), 290-306.doi.org/10.1080/00336 297.2008.10483582

Thivel, D., Isacco, L., Lazaar, N., Aucouturier, J., Ratel, S., Doré, E., Meyer, M., \& Duché, P. (2011). Effect of a 6-month school-based physical activity program on body composition and physical fitness in lean and obese schoolchildren. European Journal of Pediatrics, 170(11), 1435-1443.doi: 10.1007/s00431-011-1466-X

Tremblay, M. S., Gonzalez, S. A., Katzmarzyk, P. T., et al. (2015). Physical Activity Report Cards: Active
Healthy Kids Global Alliance and the Lancet Physical Activity Observatory. Journal of Physical Activity and Health, (12), 297- 298.

Vizcaíno, V. M., Aguilar, F. S., Gutiérrez, R. F., Martínez, M. S., López, M. S., Martínez, S. S., Garcia, E. L., \& Artalejo, F. R. (2008). Assessment of an after-school physical activity program to prevent obesity among 9-to 10-year-old children: A cluster randomized trial. International journal of Obesity, 32(1), 12-22. doi:10.1038/sj.ijo.0803738

Wickel, E.E., Eisenmann, J.C., Welk, G.J. (2009). Maturity related variation in moderate to vigorous physical activity among 9-14 year-olds. Journal of Physical Activity and Health, 6 (5), 597-605.doi. org/10.1123/jpah.6.5.597

Wong, P. C., Chia, M., Tsou, I. Y., Wansaicheong, G. K., Tan, B., Wang, J. C., Tan, J. C., Kim, C. G., Boh, G. B., \& Lim, D. (2008). Effects of a 12-week exercise training programme on aerobic fitness, body composition, blood lipids and C-reactive protein in adolescents with obesity. Annals of Academy of Medicine, 37(4), 286-293.

World Health Organization. (2006). The world health report 2006: Working together for health. World Health Organization.

Yin, Z., Gutin, B., Johnson, M. H., Hanes Jr, J., Moore, J. B., Cavnar, M., Thornburg, J., \& Barbeau, P. (2005). An environmental approach to obesity prevention in children: Medical College of Georgia FitKid Project year 1 results. Obesity Research, 13(12), 2153-2161.doi. org/10.1038/oby.2005.267

Zorba, E., Cengiz, T., \& Karacabey, K. (2011). Exercise training improves body composition, blood lipid profile and serum insulin levels in obese children. Journal of Sports Medicine and Physical Fitness, 51(4), 664-670.

Zrnzević, N., \& Zrnzević, J. (2016). Effects of teaching physical education on the morphological characteristics of male students of younger school. Glasnik Antropološkog Društva Srbije, (51), 7-16.doi: 10.5937/gads51-12155 